\title{
On the Factors Modulating the Stratocumulus to Cumulus Transitions
}

\author{
IRINA SANDU \\ Max Planck Institute for Meteorology, Hamburg, Germany, and European Centre for Medium-Range \\ Weather Forecasts, Reading, United Kingdom
}

BJORN STEVENS

Max Planck Institute for Meteorology, Hamburg, Germany

(Manuscript received 28 July 2010, in final form 5 May 2011)

\begin{abstract}
Large-eddy simulation (LES) is used to explore the role of various processes in regulating the stratocumulus to cumulus transition (SCT). Simulations are based on a composite case derived from a Lagrangian analysis of $2 \mathrm{yr}$ of data from the northeastern Pacific. The simulations reproduce well the observed transition from a compact stratocumulus layer to more broken fields of cumulus, simply as a response to increasing sea surface temperatures (SSTs) along the transition. In so doing they support earlier theoretical work that argued that the SCT was a response of boundary layer circulations to increased forcing by surface latent heat fluxes. Although the basic features of the SCT imposed by the increase in SST are robust, a variety of other factors affect the detailed character of the SCT. For example, enhanced precipitation or increased downwelling longwave radiative fluxes can accelerate the reduction in cloud cover that accompanies the SCT, while a gradual decrease in the large-scale divergence can make changes in cloud cover that accompany the SCT relatively more modest. The simulations also demonstrate that the pace of the SCT is mainly set by the strength of the temperature inversion capping the initial stratocumulus-topped boundary layer.
\end{abstract}

\section{Introduction}

The transition from unbroken sheets of stratocumulus to fields of scattered cumulus is a characteristic of cloud climatologies over the subtropical oceans. The character of this transition has been extensively studied over the past decades, both through observations (Albrecht et al. 1995; Bretherton and Pincus 1995; De Roode and Duynkerke 1997; Pincus et al. 1997) and with the help of one- or two-dimensional numerical simulations (Wang et al. 1993; Bretherton 1992; Bretherton and Wyant 1997; Bretherton et al. 1999; Krueger et al. 1995; Wyant et al. 1997; Stevens 2000). Sandu et al. (2010) took advantage of the expanding satellite record and refined meteorological reanalysis to explore the similarity among the stratocumulus to cumulus transitions (SCTs) over different ocean basins. Building on a methodology developed by Pincus et al. (1997), several datasets were used to document the evolution of the cloud and the

Corresponding author address: Irina Sandu, ECMWF, Shinfield Park, Reading RG2 9AX, United Kingdom.

E-mail: irina.sandu@ecmwf.int environmental properties along a large number of subtropical airmass trajectories. These data showed that the SCTs share essential characteristics across each of the basins, and that these characteristics support theoretical arguments developed based on past modeling (Bretherton and Wyant 1997). That is, the SCTs take place during the first three days of the trajectories (when the cloud cover decreases roughly by a factor of 2) and are mainly driven by the increase in sea surface temperatures (SSTs) along the trajectories of air parcels. This observational study also showed that the environments in which the fastest and the slowest SCTs take place are mainly distinguished by the values of lower tropospheric stability (LTS), which are primarily attributable to different values of SST. That is, the stratocumulus-topped boundary layers evolving over relatively warmer waters (and hence under weaker inversions) are prone to faster reductions in cloudiness than those evolving over colder surface waters (hence under stronger capping inversions). This suggests that the pace of the SCT is mainly controlled by the strength of the inversion capping the stratocumulustopped boundary layer at the beginning of the trajectories. 
Based on observations alone, however, it is difficult to rule out the possibility that other factors or processes may play a more important role in the cloud breakup than the increase of SSTs. Among these we can note, for instance, the changes in the downwelling longwave radiation driven by changes in the free-tropospheric humidity, the gradual reduction in the strength of largescale subsiding motion through the course of the SCT (Sandu et al. 2010), or the effects of drizzle on the boundary layer dynamics (Wang et al. 1993). Based on observations alone it is also difficult to quantify to what extent the strength of the inversion capping the stratocumulus layer sets the pace of the SCT. In this paper we use large-eddy simulation (LES) to explore these questions in more detail. Until now two obstacles have stood in the way of using LES to explore such questions. The first is that a suitably general characterization of the environmental conditions following the SCTs has not existed, and the second is the sheer computational burden of a suite of sufficiently well-resolved threedimensional simulations - this is why past work has been limited to modeling the SCT in one or two dimensions. The analysis of Sandu et al. (2010) helped overcome the first obstacle, while the general advancement of computational power (combined with advances in the treatment of radiative transfer) has made it possible to surmount the second obstacle.

The purpose of our study is twofold. First, we want to examine whether state-of-the-art LES supports ideas developed based on the previous simple numerical modeling studies and observations that the SST increase is the main driver of the SCT. Second, we want to explore how other factors that have not been accounted for in previous studies, and whose role is difficult to assess from observations, affect the SCT. Section 2 describes how the observations of Sandu et al. (2010) were used to develop a case study of the SCT. Section 3 demonstrates that the main features of the SCT-which we identify as the rapid deepening and decoupling of the boundary layer and the occurrence of shallow cumulus under the gradually thinning and dissipating stratocumulus layer-are well simulated by simply accounting for the increase in SST. Section 4 demonstrates that this basic structure of the SCT is not affected by factors such as an enhancement of the precipitation flux, a change in the large-scale divergence, or a gradual increase in downwelling longwave radiation due to the gradual humidification of the free troposphere along the airmass trajectory, although each of these factors may modulate how the SCT presents itself through the evolution of the cloud cover or the decrease in cloud albedo. Section 5 shows that the simulations capture well the observed differences between the slowest and the fastest SCTs, and demonstrates that the pace of the SCT is mainly related to the inversion strength at its starting point. Finally, section 6 discusses the controls on the cloud fraction and section 7 summarizes what we have learned through the course of our investigation.

\section{Development of a composite SCT case study}

\section{a. Methodology}

Sandu et al. (2010) showed that the characteristics of the SCT, depicted by the analysis of individual air parcel trajectories, are quite accurately reproduced by climatological (or averaged) datasets during the months of maximum cloudiness in the stratocumulus regions, especially in the northeastern Pacific. This suggests that for certain regions and time periods, averaged forcings can be considered as representative of individual trajectories and can therefore be used to initialize numerical simulations of the SCT.

Here we build on these findings to derive a case study of the SCT (referred to hereafter as our reference or REF case and described in detail in the following paragraphs). The setup of this REF case is based on a composite of the large-scale conditions encountered along a set of individual trajectories performed for the northeastern Pacific during the summer months of 2006 and 2007 [June-August (JJA)]. Both the initial profiles (liquid water potential temperature $\theta_{l}$, total water content $q_{t}$, and wind speed) and the large-scale conditions (SST and large-scale divergence), used to initialize the simulation, represent the medians of the distributions of these various properties obtained from the European Centre for Medium-Range Weather Forecasts (ECMWF) Interim Re-Analysis (ERA-Interim) (Simmons et al. 2007) for the analyzed set of trajectories [for details about the Lagrangian analysis, see section 2 of Sandu et al. (2010)].

We focus on the first three days of the trajectories only, as this is the period when the bulk of the transition in cloud cover takes place (Sandu et al. 2010). Examining the median transition lets us use observational datasets, such as Cloud-Aerosol Lidar and Infrared Pathfinder Satellite Observations (CALIPSO) and CloudSat, which might be too sparse for use in individual trajectories. We use CALIPSO to define the height of the boundary layer for the reference case, and we restrict our composites to the years in which these observations are available (i.e., 2006 and 2007), although these composites are quite similar to those obtained from larger samples [e.g., the period 2002-07 considered by Sandu et al. (2010); see Fig. 1]. 



FIG. 1. Time evolution of (a) the liquid cloud fraction and the environmental factors, (b) SST, (c) LTS, (d) large-scale divergence averaged from 1000 to $900 \mathrm{hPa}$, (e) potential temperature at $700 \mathrm{hPa}$, and (f) water vapor specific humidity at $700 \mathrm{hPa}$, during the first three days of the trajectories followed by the air parcels in the subtropical northeastern Pacific (Sandu et al. 2010). The values represent the medians of the distributions of the different properties obtained for the set of trajectories analyzed for JJA $2002-07$ (gray), for JJA 200607 (black), and for two subsets of this last set of trajectories corresponding to the slowest (dotted) and fastest (dashed) transitions in cloud fraction (see section 5 for a description). The cloud fraction is derived from MODIS level 3 data and the environmental properties from the ERA-Interim.

\section{b. Initial profiles}

As available meteorological reanalyses do not capture well the inversion strength in marine stratocumulus regions (Stevens et al. 2007), we have to reconstruct the initial profiles of $\theta_{l}$ and $q_{t}$ (Figs. 2a,b). Given that at the initial time the conditions are typical of a stratocumulustopped boundary layer, we assume the boundary layer to be well mixed. We specify the initial boundary layer depth using the mean of all CALIPSO observations during JJA 2006 and 2007 at the starting point of the median trajectory. Above this level we use a linear interpolation of the median ERA-Interim $\theta_{l}$ and $q_{t}$ profiles. We then compute the $\theta_{l}$ and $q_{t}$ values within the boundary layer so that the integrated quantities of energy and total water content for our idealized profiles are identical to the ones of the median ERA-Interim profiles. For simplicity, we use a linear interpolation of the median ERA-Interim profiles to initialize the horizontal wind components (Figs. 2c,d). Although we neglect thus the wind shear across the inversion, we do not believe that this simplification affects the conclusions of our study. The geostrophic winds are set equal to these initial wind profiles and are constant throughout the simulations.

\section{c. Large-scale conditions}

For the REF case we force the simulation by imposing the gradually increasing SST indicated by the reanalysis (Fig. 1b). Changes along the trajectory in both largescale divergence (Fig. 1d) and free-tropospheric thermodynamic state (Figs. 1e,f) are neglected, except those that result from slight imbalances between the radiative cooling and subsidence warming, or from the slight subsidence drying associated with gradients in the moisture profile. By neglecting more significant changes in the free-tropospheric state or variations in the large-scale divergence $D$ we can ask if the changing SSTs through the course of the SCT are sufficient to explain the 



FIG. 2. Median ERA-Interim profiles of (a) potential temperature (K), (b) total water specific humidity ( $\mathrm{g} \mathrm{kg}^{-1}$ ), and (c),(d) horizontal wind components $u, v\left(\mathrm{~m} \mathrm{~s}^{-1}\right)$ at the starting point of the trajectories analyzed by Sandu et al. (2010) for JJA 2006 and 2007 in the northeastern Pacific (thin lines). The superposed thick lines correspond to the idealized profiles of $\theta_{l}, q_{t}, u$, and $v$ used to initialize the reference simulation.

transition. Later we will explore how changes in other environmental properties modulate the structure of the SCT (section 4).

For the reference case, the fixed value of the largescale divergence is taken as the average over the first two days of the median ERA-Interim divergence $(1.86 \times$ $\left.10^{-6} \mathrm{~s}^{-1}\right)$. The large-scale subsidence is computed as $w_{s}=-D z$, with $z$ being the height above the ground level. Imbalances between the clear-sky radiative cooling and the subsidence warming cause less than a $0.5-\mathrm{K}$ drift in the thermal profiles through the $72 \mathrm{~h}$ of simulation (Fig. 7a). Likewise, because the free-tropospheric gradient of the $q_{t}$ profile is small, the drying of the free troposphere associated with large-scale subsidence is small and the moisture content above the inversion remains basically constant during the $72 \mathrm{~h}$ (Fig. $7 \mathrm{~b}$ ). For this reason we do not nudge the free-tropospheric profiles in order to maintain essentially constant free-tropospheric temperature (Fig. 1e) and moisture profiles.

\section{d. Simulations}

Simulations are performed with the latest version of the University of California, Los Angeles (UCLA) LES (Stevens and Seifert 2008), which has been recently used for the Rain in Cumulus over the Ocean (RICO)Global Energy and Water Cycle Experiment (GEWEX) Cloud System Study (GCSS) intercomparison case (vanZanten et al. 2011). This version differs from earlier versions (Stevens et al. 1996, 1998, 2005) through its incorporation of a fourth-order Runge-Kutta scheme instead of a blend of forward and leapfrog time differencing. The model solves prognostic equations for the velocity 
TABLE 1. Summary of simulations: domain size, averaged cloud cover (CC) during the first $48 \mathrm{~h}$ of simulation (defined as in Fig. 5), maximum cloud fraction (MaxCF) averaged over the third night, and decrease in horizontally averaged cloud albedo during the $72 \mathrm{~h}$ [computed as $\Delta A=\left(A_{0 \mathrm{~h}}-A_{72 \mathrm{~h}}\right) / A_{0 \mathrm{~h}}$ ]. The reference domain is $8.96 \times 8.96 \times 3.2 \mathrm{~km}^{3}$, while the small domain extends over $4.48 \times$ $4.48 \times 3.2 \mathrm{~km}^{3}$.

\begin{tabular}{llccc}
\hline \hline Simulation & Domain & $\overline{\mathrm{CC}}_{0-48 \mathrm{~h}}(\%)$ & $\overline{\mathrm{MaxCF}}_{3^{\text {rd }} \text { night }}(\%)$ & $\begin{array}{c}\Delta A \\
(\%)\end{array}$ \\
\hline REF & Reference & 94 & 83 & 51 \\
CST-SST & Small & 99 & 98 & 20 \\
PP & Reference & 86 & 40 & 72 \\
DIV & Reference & 94 & 88 & 38 \\
RAD & Small & 90 & 64 & 68 \\
SLOW & Reference & 97 & 87 & 44 \\
FAST & Reference & 91 & 33 & 81 \\
DTH & Small & 75 & 57 & 54 \\
DTHQT & Small & 95 & 94 & 26 \\
\hline
\end{tabular}

vector, total water mixing ratio $r_{t}$, and $\theta_{l}$, the mass mixing ratio of rainwater $r_{r}$ and the mass specific number of rainwater drops. The cloud water mixing ratio $r_{c}$ is diagnosed from $r_{t}$ and the saturation mixing ratio $r_{s}$ so that $r_{c}=\max \left(0, r_{t}-r_{r}-r_{s}\right)$. Cloud microphysical processes are treated with a two-moment bulk microphysical scheme (Seifert and Beheng 2001), which includes droplet autoconversion, accretion, drizzle, and cloud droplet sedimentation and evaporation. For all the simulations, except for the one designed to evaluate the impact of precipitation, the cloud droplet population density is fixed at $100 \mathrm{~cm}^{-3}$. Radiative processes are represented using the Monte Carlo spectral integration (McSI) method developed by Pincus and Stevens (2009), which is based on the delta-four stream radiative transfer code (Liou et al. 1988).

All of the simulations start at 1000 local time (LT) on 15 July and continue for $72 \mathrm{~h}$. The calendar day is required to properly determine the temporal evolution (diurnal cycle) of the solar zenith angle. Most simulations are performed using a domain spanning $8.96 \times 8.96 \times$ $3.2 \mathrm{~km}^{3}$, divided into $256 \times 256 \times 528$ grid points (Table 1 ). For all the simulations, the grid mesh is $35 \mathrm{~m}$ in the horizontal and $5 \mathrm{~m}$ in the vertical. Sensitivity tests have shown that the choice of this aspect ratio does not influence our results. Above $2.5 \mathrm{~km}$ the vertical grid mesh is gradually increased, with a stretching factor of $10 \%$. The boundary conditions are periodic and a sponge layer is used at the top of the domain. We note that the resolution we used is finer, and the domain as a whole is larger, than what was used in the earlier two-dimensional studies of the SCT and thus represents a considerable advance over earlier work.

Such a configuration demands a significant amount of computing time. That is, $100000 \mathrm{~h}$ of computing time are needed for a 72-h simulation. The choice of such
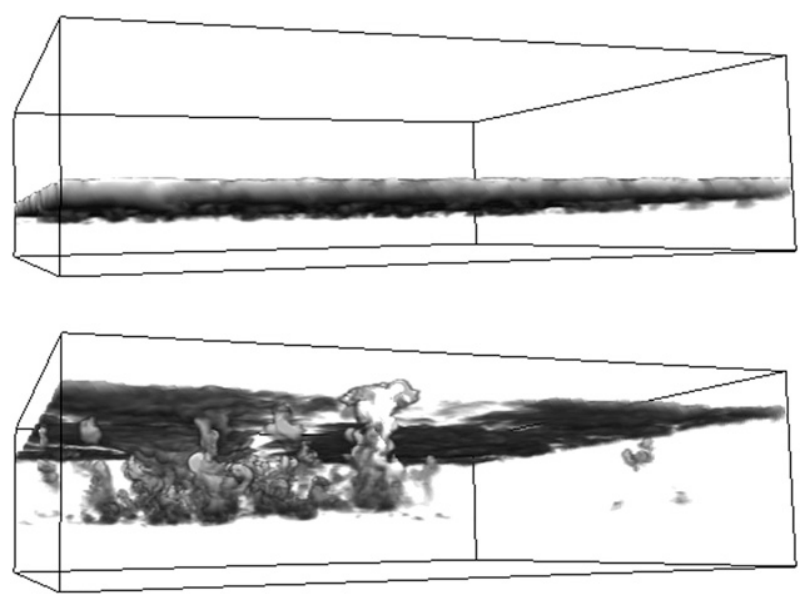

FIG. 3. An image of the cloud field simulated in the REF case, at (top) the beginning and (bottom) the end of the simulation.

a computationally demanding configuration was motivated by preliminary sensitivity tests. Coarser vertical resolution significantly affects the representation of the mixing at cloud top. A smaller horizontal domain size (e.g., $4.48 \times 4.48 \times 3.2 \mathrm{~km}^{3}$ ) has a noticeable impact in cases where precipitation plays a more important role. For weakly precipitating cases (such as the reference case), the cloud cover and albedo, however, evolve very similarly to what is simulated on the bigger (or default) domain (the difference in horizontally averaged cloud albedo at the end of the two runs is less than 5\%), despite some differences in terms of precipitation and liquid water path (LWP). In such cases we can therefore use this smaller domain for some of our sensitivity tests without missing essential information (Table 1).

\section{The SCT as a forced response to warming SSTs}

\section{a. The simulated SCT}

The REF simulation, which accounts for the increase in SST but includes none of the other observed changes in the large-scale state, reproduces well the main features of observed SCTs. Thus, daytime cloud cover is reduced by a factor of 2 over the three days of simulation, cloud-top heights roughly double, and the cloud structure transitions from a well-mixed stratocumulus regime to a cumulus regime wherein the subcloud and cloud layer are more strongly differentiated. These features of the SCT are analyzed in more detail in the following paragraphs. The flow visualizations in Fig. 3, however, give a first clear picture of the transition between the stratocumulus regime of the first morning and the cumulus regime that emerges at the end of the simulation period, as well as of the deepening of the boundary layer through the course of the SCT. 




FIG. 4. Time evolution of the cloud cover. The black dots correspond to the simulated cloud cover (computed by considering a column as cloudy for LWP $>10 \mathrm{~g} \mathrm{~m}^{-2}$ ) at the hours when MODIS observations are available (i.e., 1030 and 1330 LT). The gray dots and stars correspond to the medians of the distributions of MODIS Cloud_Fraction_Liquid and Cloud_Fraction_Day along the trajectories. These two products can be considered as the upper and lower bounds of the real cloud fraction [see appendix A of Sandu et al. (2010)].

The extent to which the simulated SCT matches the observations is difficult to evaluate more quantitatively. Because of uncertainties in measuring LWP, cloud-top heights, or cloud optical thickness (Sandu et al. 2010), the observed SCT is most easily characterized by the evolution of the cloud cover, even if we believe that its most salient feature is the structural change in the boundary layer circulation. Yet, a quantitative comparison of the simulated and observed cloud cover is also not straightforward for a couple of reasons-because it depends strongly on one's definition of a cloudy column, and also because the composite forcing does not necessarily (or precisely) lead to the composite response of the cloud cover. Still, if we use a threshold columnar LWP of $10 \mathrm{~g} \mathrm{~m}^{-2}$ (which was chosen to select cloudy columns that one could expect to see in the satellite data) to define the simulated cloud cover, there is a reasonable correspondence between the simulated cloud cover and that obtained from two different Moderate Resolution Imaging Spectroradiometer (MODIS) products at the same point in the diurnal cycle (Fig. 4).

In terms of cloud cover, the simulated SCT depends in large part on the diurnal breakup of the cloud, as nighttime cloud cover varies little over the three days of the simulation (Fig. 5). This diurnal cycle is also evident in
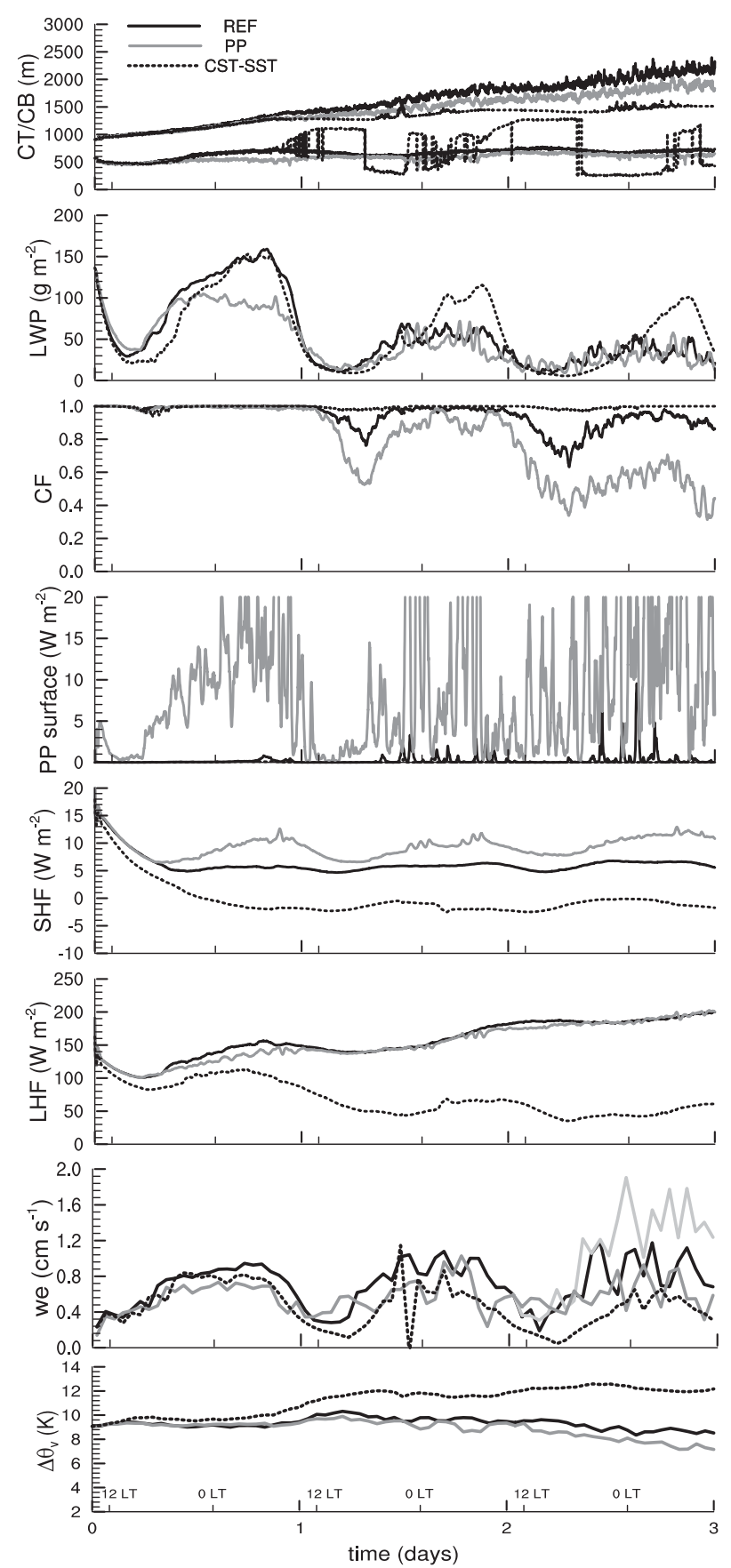

FIG. 5. Time series of the instantaneous (top to bottom) maximum and minimum cloud top and cloud base $(\mathrm{m})$, LWP $\left(\mathrm{g} \mathrm{m}^{-2}\right)$, cloud cover (a column is defined as cloudy for cloud water content $>$ $\left.0.01 \mathrm{~g} \mathrm{~kg}^{-1}\right)$, precipitation flux at the surface $\left(\mathrm{W} \mathrm{m}^{-2}\right)$, surface sensible heat flux $\left(\mathrm{W} \mathrm{m}^{-2}\right)$, surface latent heat flux $\left(\mathrm{W} \mathrm{m}^{-2}\right)$, hourly mean entrainment rates $\left(\mathrm{cm} \mathrm{s}^{-1}\right)$, and jumps in virtual potential temperature across the inversion $(\mathrm{K})$. The small tick marks on the $x$ axis indicate local noon and midnight. The black and dark gray lines correspond to the REF and PP cases, respectively. The black dotted lines correspond to the CST-SST case, and the light gray line indicates the entrainment rate in the DIV case. The values for LWP, precipitation and surface heat fluxes, entrainment rates, and $\theta_{v}$ jumps represent horizontal averages. 




FIG. 6. Time series of the hourly averages of the horizontally mean buoyancy flux profiles $\left(10^{-4} \mathrm{~m}^{2} \mathrm{~s}^{-3}\right)$ for (left) the REF and (right) the PP cases. The black line shows the zero buoyancy contour.

the observations, wherein the cloud cover at $1030 \mathrm{LT}$ is significantly higher than that at 1330 LT (Fig. 4), although the difference is perhaps not as large as in the simulations. Moreover, at certain times the evolution of the simulated cloud cover depends on the structure of a thin veil of stratocumulus that is maintained at the base of the inversion (Figs. 3 and 8 ). This is especially the case around noon on the second day and during the last $24 \mathrm{~h}$ of simulation, when the domain-averaged LWP of the stratocumulus layer ranges between 5 and $30 \mathrm{~g} \mathrm{~m}^{-2}$. Such thin cloud layers are sensitive to the representation of a variety of processes and their low amounts of liquid water render the total cloud cover sensitive to how one defines the cloud. Because the average albedo of a scene is both a more robust and a more physically meaningful indicator of the SCT than is the cloud cover, we use the relative decrease in domain-averaged cloud albedo during the $72 \mathrm{~h}$ as a metric of the amplitude of the SCT. For the REF simulation, the albedo decreases by $51 \%$, from 0.54 to 0.26 , through the course of the SCT (Table 1).

\section{b. Underlying mechanisms}

The evolution of the cloud-topped boundary layer in the REF simulation captures the transition between stratocumulus and cumulus, although the only change in environmental conditions that is included in this case is the increase in SSTs. The simplest test one can imagine in order to assert the role played by the increase of the SST in the occurrence of the SCT consists of repeating the simulation while maintaining a constant SST (i.e., the initial SST) through the course of the three days.
This sensitivity test (further referred to as CST-SST) shows that without the increase in SST the stratocumulus layer is maintained during the three days (Fig. 5) and, with the exception of one episode during the second day of simulation, no cumulus form under the stratocumulus layer (not shown). This-together with the fact that significant surface precipitation appears only after the cloud breakup (i.e., during the third day) (Fig. 5; Table 1) - suggests that our REF simulation corroborates the conceptual model of the SCT proposed by Bretherton (1992). This idea is supported by a closer analysis of the REF simulation documented through Figs. 5-8.

The central idea of Bretherton's conceptual model is that the SCT embodies a change in the structure and energetics of the boundary layer that is mainly driven by the increase in SST. The SCT arises thus as a result of the decoupling between the cloud and the subcloud layer (Figs. 6 and 7c), which is in turn mainly caused by the increasing contribution of latent heat fluxes (Fig. 5) to the energetics of the circulation as the air mass advects over progressively warmer water. These latent heat fluxes allow the cloud layer to mix with the warmer and drier free atmosphere at a rate that cannot be matched by the subcloud layer, whose dry circulations are not accelerated by latent heat release. The increasing contribution of the latent heat fluxes to the buoyancy production in the cloud layer is reflected by the fact that the nocturnal entrainment rates vary little along the simulation, despite the reduction of the cloud-top radiative cooling associated with the strong drop in LWP between the first and the third night. The persistence of high nocturnal entrainment rates 



FIG. 7. Horizontally averaged profiles of liquid water potential temperature, total water content, and buoyancy flux. In (a) and (b) the thin full and dashed lines correspond to hours 24 and 72 of REF (black), PP (dark gray), and DIV (light gray, hour 72 only) simulations. For the clarity of the plot, only the profiles corresponding to the REF simulation are shown in (c). In all panels, the thick black lines show the horizontally averaged profiles at the end of the first hour of the REF simulation.

toward the end of the simulation is also favored to some extent by the slight weakening of the inversion strength, associated with the SST increase (Fig. 5). As the buoyancy fluxes in the subcloud layer are not increasing commensurately with the ones in the cloud (Fig. 7c), negative buoyancy fluxes persist at the top of the subcloud layer. Therefore, the boundary layer is not able to recover from the diurnal decoupling on the second day and remains decoupled until the end of the simulation (Fig. 6). The idea that the SCT is mostly driven by latent heat fluxes is reinforced by the fact that $80 \%$ of the warming of the boundary layer through the course of the simulation can be attributed to the entrainment heat fluxes, which would not be the case if the sensible heat fluxes were driving the SCT.

After the cloud layer becomes decoupled from the surface on the second day, mixing with the warmer and drier free-tropospheric air entrained at cloud top continues while the moisture supply from the surface is reduced. Thereafter, the stratocumulus layer increasingly thins as its environment gradually warms and dries (Figs. $7 \mathrm{a}, \mathrm{b})$. So, superimposed on the diurnal cycle, a clear reduction in the stratocumulus layer is evident (as indicated by the very dark shadings in Fig. 8, but also by the strong decrease of the nighttime LWP between the first and the second night; Fig. 5). Meanwhile, as moisture accumulates near the surface (Fig. 7b), the subcloud layer becomes conditionally unstable relative to the cloud layer and small cumulus emerge out of it. A persistent cumulus layer, characterized by cloud fractions of $10 \%-20 \%$, develops under the dissipating stratocumulus after the first day. Although shallow cumulus supply it with moisture, the stratocumulus layer never again reaches down to near cloud base as it does during the
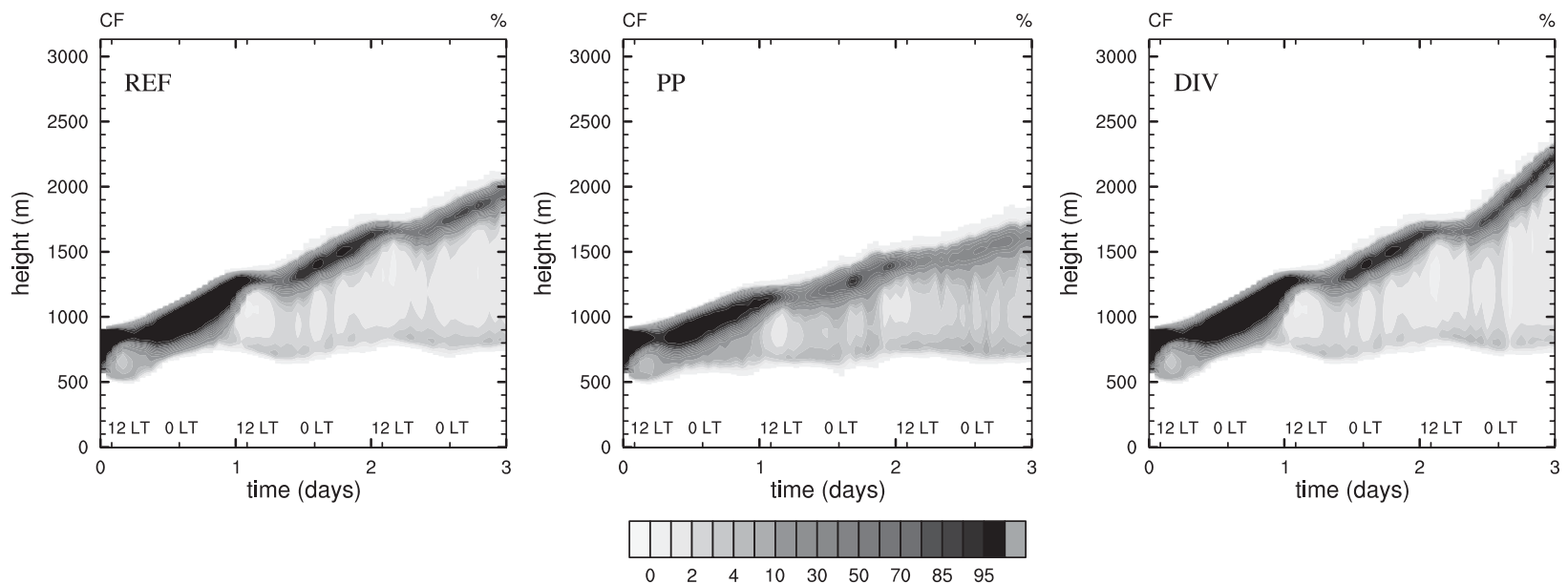

FIG. 8. Time series of the hourly averages of the cloud fraction profiles for (left) the REF, (middle) the PP, and (right) DIV cases. A grid cell is considered as cloudy for cloud water content $>0.01 \mathrm{~g} \mathrm{~kg}^{-1}$. 
first night (Fig. 8). Moreover, as the boundary layer deepens the cumulus clouds increasingly precipitate (Fig. 5 ), which in turn limits the amount of liquid water that can be transported to the stratocumulus layer, and therefore further contributes to its thinning.

\section{On some of the modulators of the SCT}

In the REF simulation we neglected some of the changes in the large-scale conditions apparent in the composite analysis of Sandu et al. (2010), most notably the reduction in the large-scale divergence on the third day and the enhancement in downwelling longwave radiative fluxes that accompanies the gradual humidification of the free troposphere through the course of the SCT (Figs. 1d,f). Through the imposition of a moderate cloud-droplet population density, precipitation does not play an important role until after cumulus convection develops (Fig. 5). Precipitation in the initial stratocumulus layer has been argued to be an essential component of the SCT (Wang et al. 1993), and the other factors we mention could also be imagined to play a role. In this section we analyze additional simulations to understand how these factors affect the main features of the SCT imposed by the increase in SST. Unless otherwise stated the setup of these simulations is identical to the one of the REF case.

\section{a. Design of the sensitivity tests}

Our first sensitivity test (referred to as PP) explores the effect of reducing the cloud droplet population density to a third of the value used in the REF case (i.e., from 100 to $33 \mathrm{~cm}^{-3}$ ). Lower cloud droplet population densities allow precipitation to develop earlier in the simulations and play a possibly more decisive role in the cloud evolution.

Our second sensitivity test (referred to as DIV) examines the effect of linearly reducing the large-scale divergence from $1.86 \times 10^{-6}$ to $0 \mathrm{~s}^{-1}$ during the last $24 \mathrm{~h}$ of simulation. This mimics the decrease in large-scale divergence indicated by the Lagrangian analysis (Fig. 1d).

Finally, our third sensitivity test (referred to as RAD) investigates whether the SCT is affected by a gradual increase in the downwelling longwave radiation that accompanies the gradual moistening of the upper troposphere during the $72 \mathrm{~h}$ (Fig. 1f). We note that increasing the amount of $\mathrm{CO}_{2}$ would have a similar effect, a manifestation of what some refer to as the indirect effect of $\mathrm{CO}_{2}$ on climate through an influence on cloudiness that is not modulated by surface temperature changes. The special case where the moistening of the free troposphere extends to the top of the boundary layer, thereby affecting the properties of the entrained air, is explored in the next section. To realize the RAD simulation we modify the downwelling radiation by gradually moistening the free troposphere above the simulation domain. The downwelling longwave radiation increases by $10 \mathrm{~W} \mathrm{~m}^{-2}$ during the $72 \mathrm{~h}$ of simulation, which roughly corresponds to increasing the total water mixing ratio by $2.5 \mathrm{~g} \mathrm{~kg}^{-1}$ above $2500 \mathrm{~m}$. Because the development of precipitation is not important in the RAD case, the simulation is performed on the smaller domain mentioned in section 2d (Table 1).

\section{b. Modulation of the SCT amplitude}

The factors we considered (divergence, radiation, precipitation) do not change the basic character of the SCT: each simulation retains the essential features of a systematic growth of the boundary layer, accompanied by a decoupling of the stratocumulus from the subcloud layer, a gradual thinning and dissipation of the stratocumulus layer, the occurrence of shallow cumulus underneath, and a sharp reduction in daytime albedo over the simulation period. However, they do shape aspects of the character of the transition, particularly the cloud amount. The main difference between the three sensitivity tests and the reference case is apparent in the structure of the thin stratocumulus layer that lingers atop the cumulus layer after the layers decouple. The amplitude of the SCT as measured by the values of the cloud cover during the last night of simulation and by the relative change in albedo during the three days for the various simulations is provided in Table 1. Precipitation and even the small changes in the downwelling longwave radiation amplify the transition in cloud fraction by more strongly depleting the stratocumulus layer. The decrease in largescale divergence (perhaps surprisingly) helps maintaining a thicker and less broken stratocumulus layer. The effects of various processes on cloud cover are well spanned by the PP and DIV cases, whose cloud field is visualized along with that of the REF simulation at the end of the third day (Fig. 9).

The amplitude of the SCT is the most significantly affected by the enhancement of precipitation imposed in PP case (Figs. 5 and 8; Table 1). Precipitation was shown to affect the evolution of cloud-top boundary layers in a number of ways, namely by limiting their growth (Stevens et al. 1998; Stevens 2007) (via reducing the cloud-top entrainment) and by modifying their mixing state (Lu and Seinfeld 2005; Sandu et al. 2008). These effects act in our case to maintain a shallower (Fig. 5) and more persistently decoupled boundary layer through the entirety of the simulation (Fig. 6). Unlike in the REF case, where the stratocumulus precipitates much less, the initial diurnal decoupling is in this case 

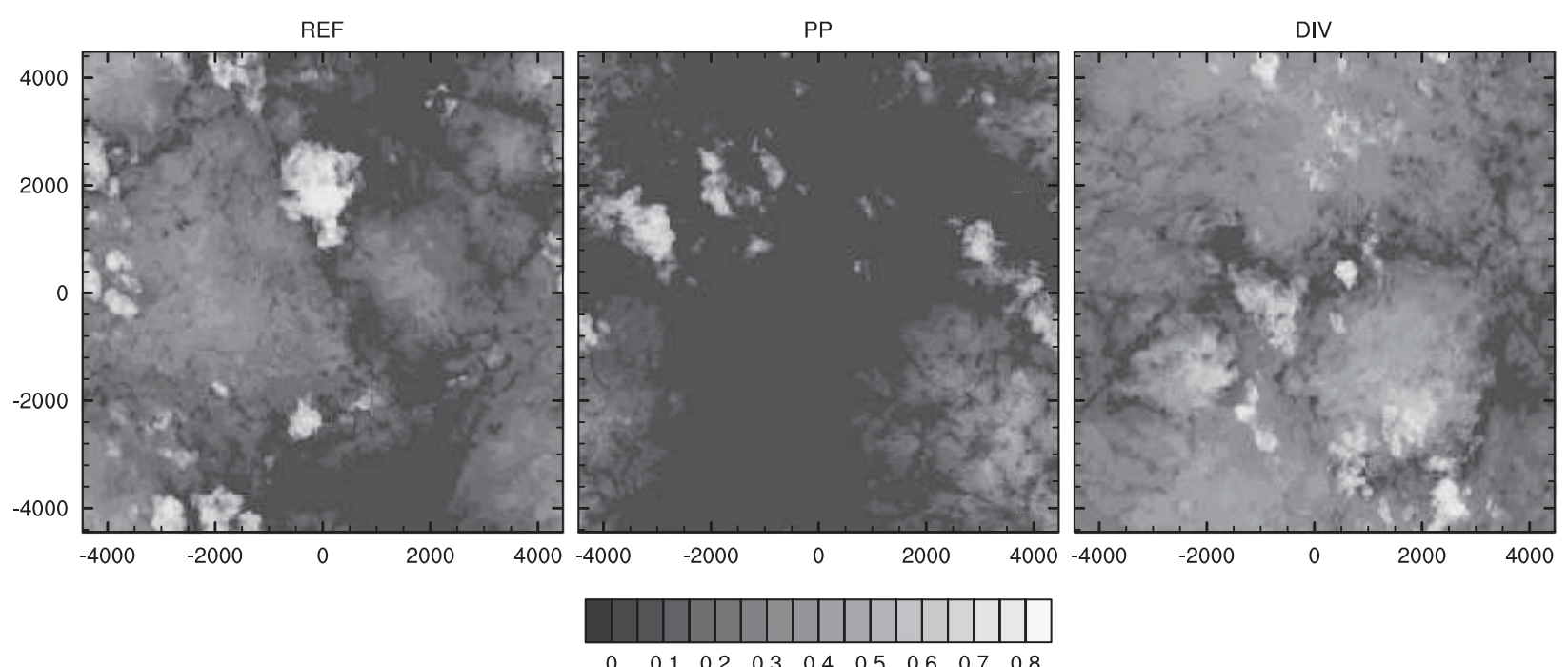

$\begin{array}{lllllllll}0 & 0.1 & 0.2 & 0.3 & 0.4 & 0.5 & 0.6 & 0.7 & 0.8\end{array}$

FIG. 9. Snapshots of cloud albedo at the end of (left) REF, (middle) PP, and (right) DIV simulations. The cloud field simulated in RAD case is not shown but closely resembles the one simulated in PP.

maintained throughout the first night of simulation by the evaporation of rain drops, which moistens and cools the subcloud layer (Figs. 7a,b), thereby stabilizing the layer. The weakening of the water vapor transport from the surface toward the cloud layer, together with the depletion of cloud water via precipitation, limits the stratocumulus reformation during the three nights (Fig. 8; Table 1). The moistening and cooling of the subcloud layer also affects somewhat the underlying shallow cumulus layer. As the air above the surface is cooler, the surface sensible heat flux increases compared to the REF run (Fig. 5). So it reinforces the convective motions in the surface layer (Fig. 6) and thereby promotes an earlier formation of shallow cumulus (Fig. 8). In the meantime, as the cumulus clouds develop in a moister and colder layer (600-900 m; Figs. 7a,b), they are more frequent/persistent than in the REF case (Fig. 8).

The decrease in the large-scale divergence leads to a less pronounced SCT, as the stratocumulus cloud topping the cumulus layer dissipates less in the DIV case relative to the other cases (Figs. 8 and 9; Table 1). For a boundary layer depth $h$, we expect

$$
\frac{\partial h}{\partial t}=E-D h
$$

where $E$ is the entrainment rate and $D h$ is the subsidence velocity, with $D$ being the divergence. Hence for a constant, or bigger, entrainment rate, weaker divergence implies a deeper boundary layer. As the boundary layer deepens more than in the REF case, for similar entrainment rates (Fig. 5) and similar profiles of temperature and total water content (not shown), the stratocumulus becomes thicker during the first half of the third day of the DIV simulation. As its liquid water content is bigger, the cloud-top radiative cooling (and hence the entrainment rate) becomes stronger than in the REF case during the last night of simulation (Fig. 5). The boundary layer increases thus even more rapidly, and although it becomes slightly warmer and drier in its upper part (Figs. 7a,b), the stratocumulus can thicken more than in the REF case.

Even a small increase in downwelling longwave radiation (associated with the moistening of the free troposphere) appears to significantly impact the evolution of the stratocumulus layer during the third day of simulation (Table 1). Mechanistically this can be understood because as the downwelling longwave flux is increased, the net flux divergence at cloud top is reduced. During the first half of the RAD simulation, the imposed diminution of the cloud-top radiative cooling is very small (downwelling longwave fluxes increase by only about $3-5 \mathrm{~W} \mathrm{~m}^{-2}$ and thus reduce radiative cooling rates by only about $4 \%-7 \%$ ) and the effect is relatively small. However, by the third day downwelling longwave fluxes have increased sufficiently to reduce the cloud-top longwave flux divergence by as much as $7-10 \mathrm{~W} \mathrm{~m}^{-2}$ and this begins to have a significant impact on the structure of the more delicate stratocumulus layer present during this period.

These sensitivity tests show thus that although precipitation or changes in the large-scale divergence or downwelling longwave radiation along the SCT do not affect the main features of the transition, they can modulate some of its specific features, particularly the cloud amount in the decoupled state. These changes reflect the delicacy of the lingering stratocumulus layer and its 
susceptibility to perturbation from a variety of processes. So while such changes may be important to quantitative descriptions of the SCT (and hence more detailed comparisons to data), they are not necessary to explain its basic features. Moreover, their impact on the SCT's amplitude depends on whether the environmental conditions favor the persistence of a thin stratocumulus layer under the inversion till the end of the simulation. That is, if the stratocumulus layer was to disappear at the end of the second day of simulation in the reference case, the sensitivity of the SCT's amplitude to the factors we considered would diminish.

\section{What sets the time scale of the SCT?}

So far our simulations confirmed that the SCT can emerge from a dynamical response of the system to the gradual increase in SST. Furthermore, other factors whose role in the process is not clear from the observations are not necessary to explain the basic structure of the SCT, but they may help set some of its particular features, particularly the cloud amount. A last aspect that we would like to better understand is what controls the time scale over which the SCT is realized. The observational study of Sandu et al. (2010) showed that the environments in which the slowest and respectively the fastest SCTs take place are mainly distinguished by their values of LTS, which are primarily attributable to different values of SST [see Figs. 4 and 5 of Sandu et al. (2010) and our Fig. 1]. Sandu et al.'s observations suggest that the pace of the transition is mainly controlled by the strength of the temperature inversion capping the boundary layer, for which the LTS is often used as a proxy. In this section we investigate this hypothesis more quantitatively by making use of additional simulations.

As a first step, we derive two variations of the reference case, corresponding to a slower and a faster SCT, and we demonstrate that LES can capture the observed differences between the two cases (section 5a). We then examine whether the difference in the inversion strength is indeed the primary cause for the differences between the slow and the fast SCTs (section $5 b$ ).

\section{a. Slow versus fast SCTs}

\section{1) SEtup of The Simulations}

The two cases corresponding to a slower and a faster transition in cloud fraction are derived similarly to the REF case and hereafter will be referred to as the SLOW and FAST cases. The initial profiles and large-scale forcings (Figs. 10 and 1) represent the medians of the distributions obtained for $25 \%$ of the total number of trajectories analyzed for the northeastern Pacific during
JJA 2006-07 that are characterized respectively by the largest and the smallest average cloud fraction over their first $48 \mathrm{~h}$ (Fig. 1a). The large-scale divergence follows its prescription for the REF case.

The two sets of initial conditions (Figs. 1 and 10) differ in many aspects: SST values, inversion strength, boundary layer mean state, free-tropospheric $\theta_{l}$ and $q_{t}$ (both in terms of absolute values and gradients), and wind speeds all differ. The initial boundary layer depth is also slightly different, as the boundary layer is $50 \mathrm{~m}$ shallower (deeper) in the SLOW (FAST) cases compared to the REF case. We infer these changes in boundary layer depth from dense MODIS samples rather than sparser (but more accurate) CALIPSO observations in order to reduce the sampling noise.

\section{2) DifFERENCES BETWEEN THE TWO CASES}

LES reproduces well the observed differences between the two cases (Figs. 11 and 12). In the SLOW case a thin but almost unbroken stratocumulus deck tops the shallow cumulus field until the end of the simulation, while in the FAST case the stratocumulus deck dissipates more efficiently (as demonstrated by the lower average cloud cover over the first two days; see Table 1 and Fig. 12) and it almost disappears during the third day. This behavior is also well illustrated by the average over the last night of simulation of the maximum cloud fraction, and by the overall decrease in cloud albedo obtained in the two cases (Table 1).

The first $24 \mathrm{~h}$ are crucial for the diverging evolution of the SLOW and FAST simulations, as the most striking differences occur during this period, both in terms of the growth rate (Fig. 11; Table 2) and the mixing state of the boundary layer (Fig. 13). Differences in boundary layer growth rates may arise from the differences in the inversion strength (Figs. 10,11, and 1c), the surface latent heat flux (LHF) (Fig. 11), and/or differences in the radiative forcing arising from differences in the freetropospheric moisture (Fig. 10) between the two cases. That is, the more pronounced deepening of the boundary layer in the FAST case is favored both by the weaker temperature inversion, and by the stronger latent heat fluxes. The moister free troposphere that characterizes the FAST case (Fig. 10b) acts to limit the difference in boundary layer growth rate between the two simulations (by decreasing the cloud-top longwave flux divergence by $10 \mathrm{~W} \mathrm{~m}^{-2}$ compared to the SLOW case).

The different boundary layer depths are accompanied by a different evolution of the mean state during the first $24 \mathrm{~h}$ of the two simulations. Thus, the deeper boundary layer remains decoupled during the first night of simulation (Fig. 13, FAST case), while the shallower one becomes rapidly well mixed after the initial diurnal 

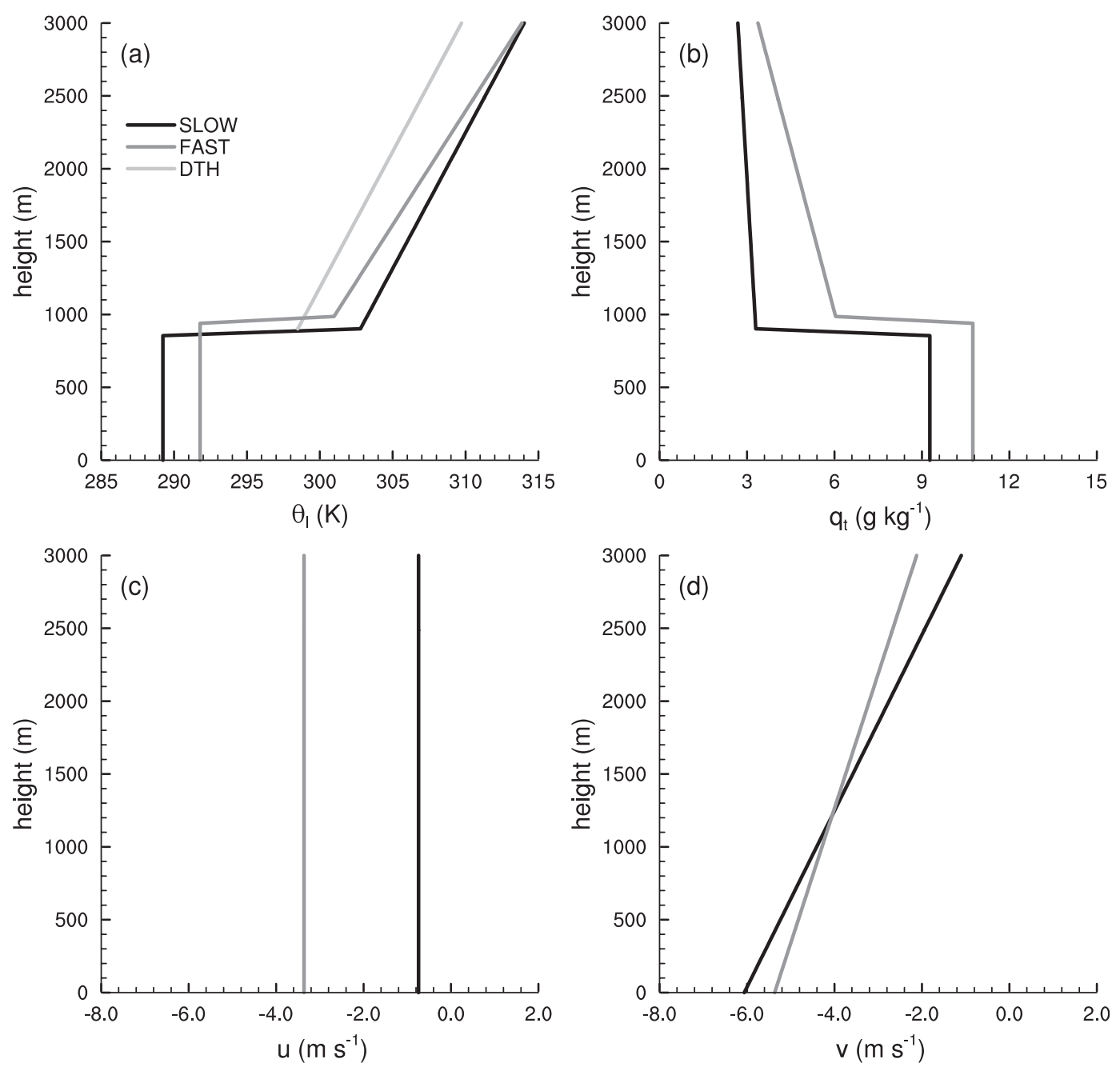

FIG. 10. Idealized profiles of (a) liquid water potential temperature, (b) total water content, and (c) $u$ and (d) $v$ wind components used to initialize the SLOW (black) and FAST (dark gray) cases. The free-tropospheric profile of $\theta_{l}$ used to initialize the DTH simulation is shown in light gray.

decoupling (Fig. 13, SLOW case). These differences further result in a different subsequent evolution of the thermodynamic state of the two boundary layers. Thus, the water vapor supply from the surface continuously accumulates in the lower part of the boundary layer in the FAST case, while in the SLOW case it is more efficiently transported into the stratocumulus layer. As a result, after the first $24 \mathrm{~h}$, the initial difference in water content between the FAST and SLOW simulations (Fig. $10 \mathrm{~b}$ ) is magnified in the lower part of the boundary layer but is less evident in its upper part (Fig. 14b). Meanwhile, the upper part of the boundary layer simulated in the FAST case becomes increasingly warmer than the one simulated in the SLOW case (Fig. 14a). Consequently, as it evolves in an environment with a similar (or slightly bigger) water content, but with a considerably higher temperature (Fig. 14), the cloud layer simulated in the
FAST case thins and breaks up more rapidly (Figs. 11 and 12; Table 1). As the stratocumulus thins during the last part of the simulation, the decrease in cloud-top radiative cooling associated with the moister free troposphere typical for the FAST case becomes increasingly more important and acts to further amplify the cloud thinning and breakup compared to the SLOW case (according to our findings in section 4).

\section{b. Role of the inversion strength}

In a number of simulations whose results we do not belabor, we explored the influence of the various factors on the time scale of the SCT and found the initial inversion strength to be decisive. This point is demonstrated by a simulation we call DTH, in which we repeat the SLOW simulation with a modified free-tropospheric $\theta_{l}$ so that the jump across the inversion becomes identical 



FIG. 11. As in Fig. 5, but for the SLOW (black) and FAST (dark gray) cases.

to that of the FAST case (Fig. 10a), while the other conditions are kept unchanged. The DTH and the FAST simulations are thus subject to the same inversion strength but are characterized by all the other differences between the SLOW and FAST cases described above. We use $\Delta \theta_{l}$ (which is quite close to $\Delta \theta_{v}$ ) rather than LTS to characterize the inversion strength because in our case the LTS difference (Fig. 1c) does not capture well the difference in inversion strength between the two cases because of significant differences in the gradients of $\theta_{l}$ within the free troposphere (Fig. 10a).

Reducing the inversion strength causes a strong increase of the first 24-h boundary layer growth rate, an increase that almost equals that obtained for the FAST case (Table 2). The SST, wind speed, and initial $q_{t}$ profile are the same, so the latent heat fluxes evolve similarly in the DTH and SLOW cases (not shown) and therefore contribute by the same amount to the boundary layer growth. The cloud-top radiative cooling, which can also affect the boundary layer deepening, is also basically the same in the two simulations (the decrease in freetropospheric temperature imposed in the DTH case does not have a significant effect on the downwelling longwave radiation). This suggests that the inversion strength plays the crucial role in setting the boundary layer growth rate during the decisive first $24 \mathrm{~h}$.

The more rapid deepening of the boundary layer in the DTH relative to the SLOW case during the first $24 \mathrm{~h}$ leads to differences in the subsequent evolution of the cloud layer. These differences resemble differences between the FAST and SLOW case (Table 1; see the notably stronger decrease in averaged cloud cover during the first $48 \mathrm{~h}$ ). In other words, the time scale over which the SCT is realized is reduced by simply changing its initial inversion strength. The reasoning is that a deeper boundary layer results from more cloud-top entrainment, which enhances (particularly from the point of view of the water budget) the differentiation between the cloud and subcloud layer (Fig. 14). The upper part of the boundary layer thus becomes drier than the one simulated in the SLOW case, while the $\theta_{l}$ is about the same (Fig. 14). As a result, the stratocumulus layer is more difficult to sustain than in the SLOW case.

The idea that the inversion strength is decisive is consistent with the finding that the most substantial differences in terms of boundary layer growth rate between the SLOW and FAST cases appear during the first $24 \mathrm{~h}$ (Table 2). Unlike differences in the other factors, the differences in the inversion strength are most pronounced at the beginning of the simulations (Fig. 11). Because the gradient in the free-tropospheric $\theta_{l}$ is larger in the FAST case, the inversion strength tends to increase more than in the SLOW case as the boundary layer deepens, thereby moderating differences in the inversion strength as time goes on. This is likely one of the reasons why the growth rate of the boundary layer at the end of the SLOW case is more commensurate with (actually somewhat larger than) the FAST case (Table 2 ). The other reason for the slightly stronger entrainment rate/boundary layer growth rate obtained (on 


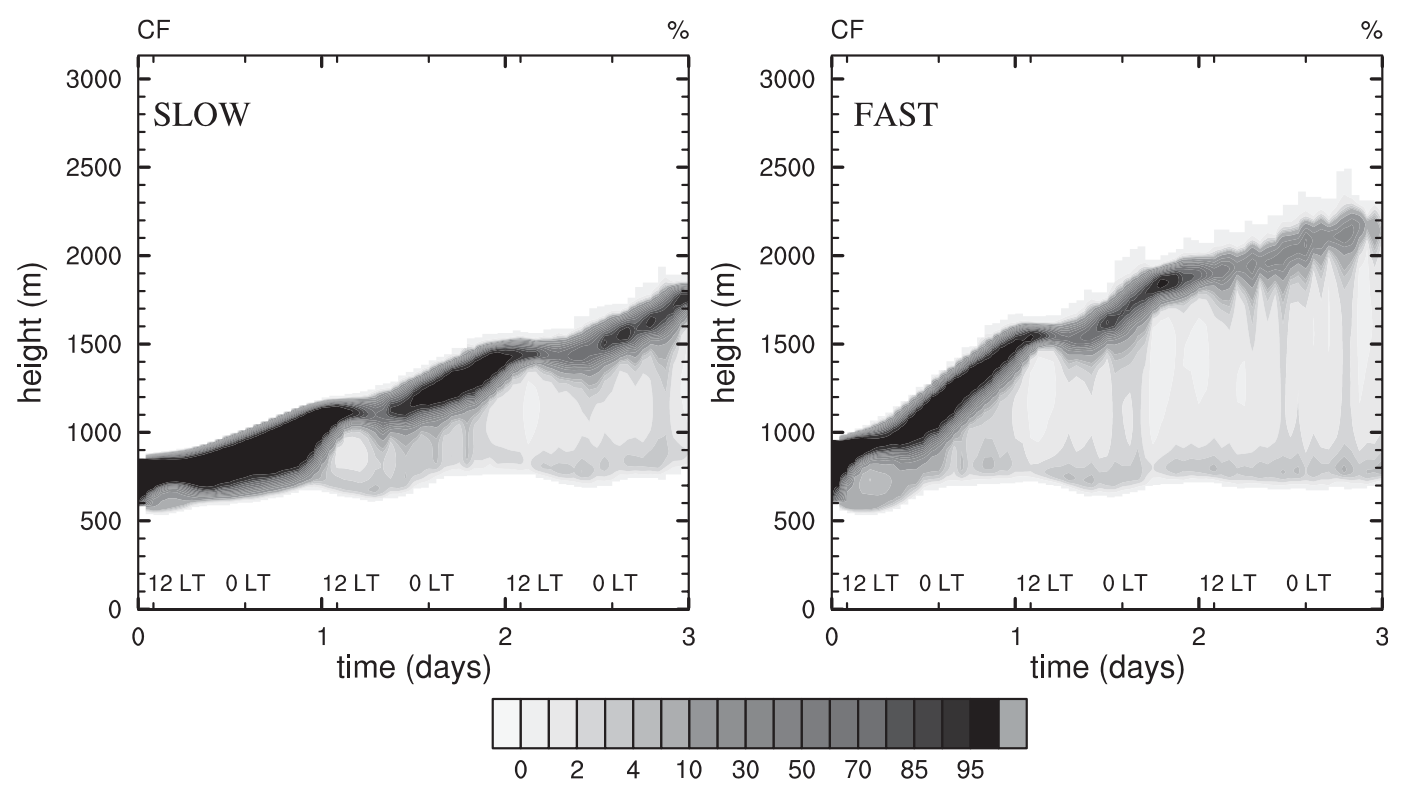

FIG. 12. As in Fig. 8, but for the SLOW and FAST cases.

average) in the last part of the SLOW simulation is the more substantial cloud-top radiative cooling, associated with the thicker stratocumulus layer (Fig. 12).

The primary importance of the inversion strength for the pace of the SCT is further supported by a couple of other tests we have performed. These include repeating the DTH simulation with increased free-tropospheric moisture so that the jump in $q_{t}$ across the inversion is identical to the one of the FAST case (DTHQT case). It appeared that during the first $24 \mathrm{~h}$ of this simulation, the growth rate of the boundary layer was identical to the one obtained in the DTH case (Table 2). This suggests that the effect of increasing the free-tropospheric moisture on the boundary layer growth rate (via its impacts on the cloud-top radiative cooling and the mixing at cloud top) is small compared to the effect of reducing the inversion strength (the $\theta_{v}$ jumps at cloud top are nearly identical in the DTH, DTHQT, and FAST simulations). Over a longer term, the moistening of the free troposphere reduces the efficiency of the stratocumulus evaporation via mixing with the free-tropospheric air, so that the stratocumulus layer dissipates less than in the DTH case (Table 1). This result is not unexpected and corroborates previous work showing how boundary layer clouds are affected if they grow in moister environments (Stevens 2007). Finally, another test consisted of repeating the SLOW simulation with increased wind speeds, identical to those considered in the FAST case. Differences in wind speed between the two cases are modest and are also not able to explain the differences between the two SCTs (not shown).

\section{Controls on cloud fraction}

The simulations have shown the cloud cover to be quite sensitive to a number of details in the specification of our case study. Moeng (2000) found that the cloud-top entrainment instability parameter $\kappa=1+\Delta \theta_{l} /\left(L / C_{p}\right) \Delta q_{t}$ effectively ordered cloud fraction for a wide range of simulations, with a sharp reduction in cloud fraction associated with values of $\kappa$ larger than the critical value required for buoyancy reversal to be possible. More recently Lock (2009) performed a similar analysis and independently found similar behavior. In this section we look across our full suite of simulations to explore whether or not this organizing principle can explain changes seen in our simulations.

In Fig. 15 we plot cloud cover versus $\kappa$ for all of our simulations. The sharp reduction in cloud cover as $\kappa$ increases is readily apparent. Cloud cover is universally large when $\kappa<0.2$ and universally small when $\kappa>0.4$, in broad agreement with previous studies. However, there is considerable scatter in the value of cloud cover over intermediate values of $\kappa$, and our simulations support

TABLE 2. Growth rate of the boundary layer in the SLOW, FAST, DTH, and DTHQT cases during the periods $12-24 \mathrm{~h}$ and 60-72 h.

\begin{tabular}{lcc}
\hline \hline Simulation & $12-24 \mathrm{~h}(\mathrm{~m})$ & $60-72 \mathrm{~h}(\mathrm{~m})$ \\
\hline SLOW & 200 & 231 \\
FAST & 379 & 121 \\
DTH & 331 & 306 \\
DTHDQ & 331 & 352 \\
\hline
\end{tabular}




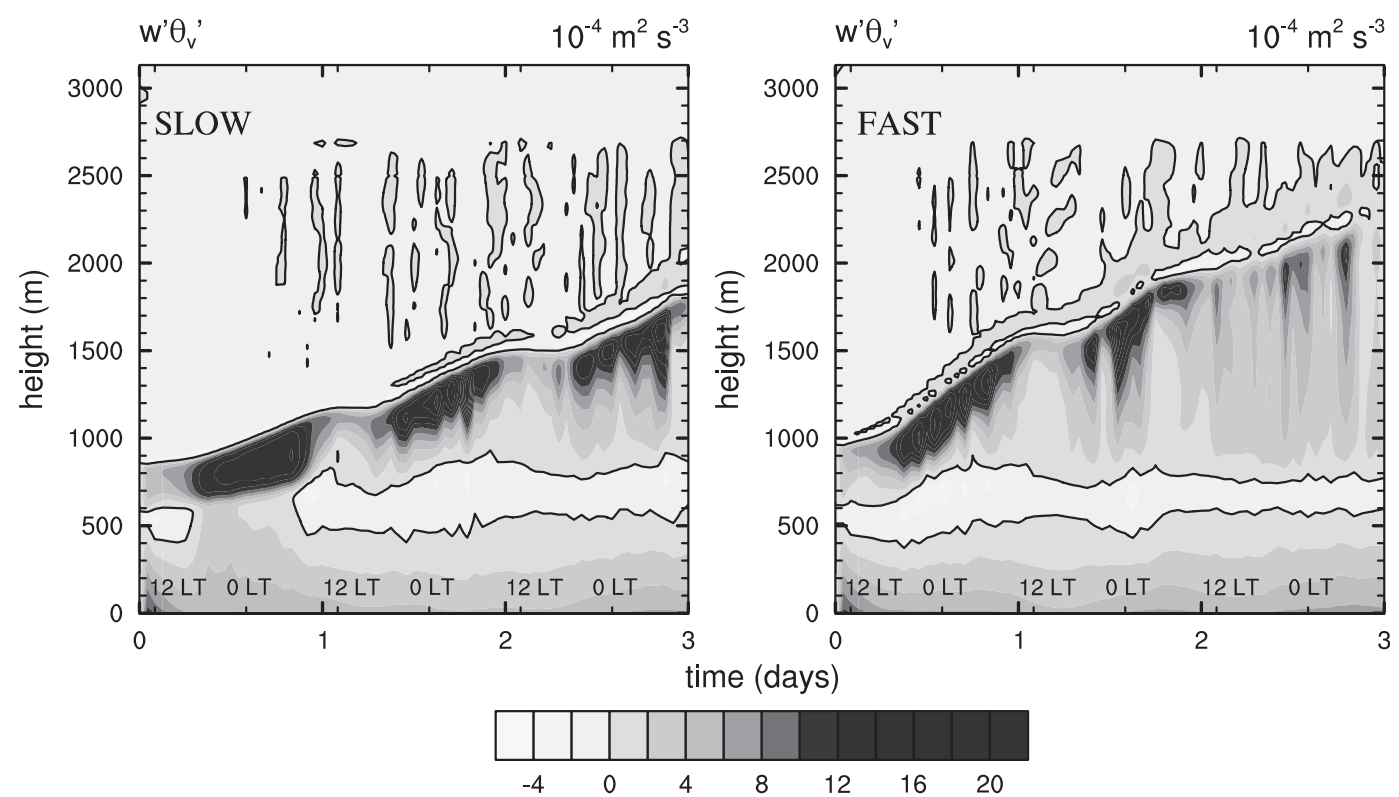

FIG. 13. As in Fig. 6, but for the SLOW and FAST cases.

neither a threshold in cloud cover associated with a critical value of $\kappa$ nor a one-to-one relationship between $\kappa$ and cloud cover. That cloud cover should generally decrease as $\kappa$ increases is reasonable, as larger values of $\kappa$ imply the entrainment of relatively drier air. The relative scatter, however, indicates that other processes also play an important role.

That other studies find a critical value of kappa near the buoyancy reversal limit may simply reflect that under conditions that permit buoyancy reversal, mixing in the LES can be enhanced in proportion to the effective diffusivity of the model, which is set by a combination of the numerical methods employed and the vertical grid spacing (Mellado 2010). Hence in conditions that support buoyancy reversal, LES is likely to overestimate cloud-top mixing and to do so preferentially for conditions that involve mixtures with unusually dry air, thereby leading to a sharp reduction in cloud amount, a reduction we believe is enhanced by numerical artifacts in the representation of cloud-top mixing by LES. Because both Moeng (2000) and Lock (2009) use somewhat coarser grids than those employed in the present study,


FIG. 14. Horizontally averaged profiles of (a) liquid water potential temperature and (b) total water content for the SLOW (black), FAST (dark gray), and DTH (light gray) simulations. The solid and dashed lines correspond to hours 24 and 72, respectively. 




FIG. 15. Hourly averaged cloud cover (defined as in Fig. 5) against the hourly averages of the $\kappa$ parameter as defined in Moeng (2000)—that is, $\kappa=1+\Delta \theta_{l} /\left(L / C_{p}\right) \Delta q_{l}$-for all the simulations.

we expect this effect to be exaggerated in their studies relative to the present work. As the entrainment processes become even better represented by LES, we thus expect the scatter in cloud fraction as a function of $\kappa$ to only increase. This analysis only reinforces the delicacy of cloud cover to both physical processes and details of the mean state and helps us understand why robust parameterizations of cloud cover remain elusive.

\section{Conclusions}

Large-eddy simulation (LES) of the marine atmospheric boundary layer, initialized and forced using data that characterizes the environment along the stratocumulus to cumulus transition (SCT) represents well the major features of this observed transition. Not only do the simulations reproduce the main features of observed SCT, but they are also able to differentiate between conditions that in the observations appear to favor a rapid transition and those that appear to favor a slower transition. This suggests that such an approach is useful for gaining further insights about the factors modulating the shift in cloud regimes, from persistent and well-mixed stratocumulus at the start of the SCT to broken fields of cumulus at the end of the SCT.

Our simulations confirm that the main features of observed SCTs can be reproduced simply by accounting for variations in the sea surface temperature (SST) along the transition (and neglecting variations in other factors), which supports the conceptual model developed by Bretherton (1992). They strengthen thus the idea that the increasing SST is the driving force for the SCT.

By varying basic parameters of our observationally based case study of the SCT we were able to investigate how various factors, whose role in the cloud breakup is hard to assess from observations alone and which were not examined by previous modeling studies, affect the SCT. Factors such as an enhancement of precipitation, a decrease in the large-scale divergence, or a small increase in the downwelling longwave radiation along the transition are shown to play a quantitative, but not qualitative, role in the evolution of the SCT, whose basic structure is set by the increase in SSTs. A decrease in the divergence, for example, can weaken the cloud breakup, while enhanced precipitation, as well as the gradual reduction in radiative forcing accompanying the moistening of the free troposphere (or increasing concentrations of $\mathrm{CO}_{2}$ for that matter), may amplify it. We also use LES to demonstrate that the time scale of the SCT is mostly determined by the strength of the temperature inversion capping the stratocumulus-topped boundary layer.

Our study sheds thus more light on the factors controlling the SCT and demonstrates once more how sensitive these boundary layer clouds, particularly the cloud cover, are to subtle changes in the large-scale conditions. Moreover, it also sets up an interesting model evaluation framework as it focuses on transitions in cloudiness and boundary layer structure. This constitutes the object of a current intercomparison study of both LES and single column models within the Boundary Layer Clouds Working Group of the GEWEX Cloud System Studies.

Acknowledgments. ECMWF ERA-Interim data used in this study have been obtained from the ECMWF data server (http://www.ecmwf.int/products/data/). The simulations were performed using the facilities of the Deutsches Klimarechenzentrum. We also acknowledge Stephan de Roode, Pier Siebesma, Suvarchal K. Cheedela, and Robert Pincus for the enlightening discussions about this work, as well as three anonymous reviewers for their valuable comments. This work was supported by the Alexander von Humboldt foundation and the Max Planck Institute for Meteorology.

\section{REFERENCES}

Albrecht, B. A., C. S. Bretherton, D. W. Johnson, W. H. Schubert, and A. S. Frisch, 1995: The Atlantic Stratocumulus Transition Experiment-ASTEX. Bull. Amer. Meteor. Soc., 76, 889-904.

Bretherton, C. S., 1992: A conceptual model of the stratocumulustrade-cumulus transition in the subtropical oceans. Proc. 11th Int. Conf. on Clouds and Precipitation, Montreal, QC, Canada, ICCP, 374-377. 
and R. Pincus, 1995: Cloudiness and marine boundary layer dynamics in the ASTEX Lagrangian experiments. Part I: Synoptic setting and vertical structure. J. Atmos. Sci., 52, 2707-2723.

— stability, and decoupling of cloud-topped boundary layers. J. Atmos. Sci., 54, 148-167.

—, S. K. Krueger, M. C. Wyant, P. Bechtold, E. van Meijgaard, B. Stevens, and J. Teixeira, 1999: A GCSS boundary-layer cloud model intercomparison study of the first ASTEX Lagrangian experiment. Bound.-Layer Meteor., 93, 341-380.

De Roode, S., and P. Duynkerke, 1997: Observed Lagrangian transition of stratocumulus into cumulus during ASTEX: Mean state and turbulence structure. J. Atmos. Sci., 54, 2157-2173.

Krueger, S. K., G. T. McLean, and Q. Fu, 1995: Numerical simulation of the stratus-to-cumulus transition in the subtropical marine boundary layer. Part I: Boundary-layer structure. J. Atmos. Sci., 52, 2839-2850.

Liou, K.-N., Q. Fu, and T. Ackerman, 1988: A simple formulation of the delta-four-stream approximation for radiative transfer parameterizations. J. Atmos. Sci., 45, 1940-1948.

Lock, A., 2009: Factors influencing cloud area at the capping inversion for shallow cumulus clouds. Quart. J. Roy. Meteor. Soc., 135, 941-952.

Lu, M. L., and J. H. Seinfeld, 2005: Study of the aerosol indirect effect by large-eddy simulation of marine stratocumulus. J. Atmos. Sci., 62, 3909-3932.

Mellado, J., 2010: The evaporatively driven cloud-top mixing layer. J. Fluid Mech., 660, 5-36.

Moeng, C.-H., 2000: Entrainment rate, cloud fraction, and liquid water path of PBL stratocumulus clouds. J. Atmos. Sci., 57, 3627-3643.

Pincus, R., and B. Stevens, 2009: Monte Carlo spectral integration: A consistent approximation for radiative transfer in large eddy simulations. J. Adv. Model. Earth Syst., 1 (1), doi:10.3894/ JAMES.2009.1.1.

_ - M. Baker, and C. Bretherton, 1997: What controls stratocumulus radiation properties? Lagrangian observations of cloud evolution. J. Atmos. Sci., 54, 2215-2236.

Sandu, I., J. L. Brenguier, O. Geoffroy, O. Thouron, and V. Masson, 2008: Aerosol impacts on the diurnal cycle of marine stratocumulus. J. Atmos. Sci., 65, 2705-2718.
B. Stevens, and R. Pincus, 2010: On the transitions in marine boundary layer cloudiness. Atmos. Chem. Phys., 10, 2377-2391.

Seifert, A., and K. Beheng, 2001: A double-moment parameterization for simulating autoconversion, accretion and self-collection. Atmos. Res., 59-60, 265-281.

Simmons, A., S. Uppala, D. Dee, and S. Kobayashi, 2007: EraInterim: New ECMWF reanalysis products from 1989 onwards. ECMWF Newsletter, No. 110, ECMWF, Reading, United Kingdom, 25-35. [Available online at http://www.ecmwf.int/ publications/newsletters/pdf/110_rev.pdf.]

Stevens, B., 2000: Cloud transitions and decoupling in shear-free stratocumulus-topped boundary layers. Geophys. Res. Lett., 27, 2557-2560.

, 2007: On the growth of layers of non-precipitating cumulus convection. J. Atmos. Sci., 64, 2916-2931.

— of microphysical choices in simulations of shallow cumulus convection. J. Meteor. Soc. Japan, 86, 143-162.

—, G. Feingold, W. C. Cotton, and R. L. Walko, 1996: Elements of the microphysical structure of numerically simulated nonprecipitating stratocumulus. J. Atmos. Sci., 53, 980-1006.

— W. C. Cotton, G. Feingold, and C.-H. Moeng, 1998: Largeeddy simulations strongly precipitating, shallow, stratocumulustopped boundary layers. J. Atmos. Sci., 55, 3616-3638.

— , and Coauthors, 2005: Evaluation of large-eddy simulations via observations of nocturnal marine stratocumulus. Mon. Wea. Rev., 133, 1443-1462.

—, A. Beljaars, S. Bordoni, C. Holloway, M. Koehler, S. Krueger, V. Savic-Jovcic, and Y. Zhang, 2007: On the structure of the lower troposphere in the summertime stratocumulus regime of the northeast Pacific. Mon. Wea. Rev., 135, 985-1005.

vanZanten, M. C., and Coauthors, 2011: Controls on precipitation and cloudiness in simulations of trade-wind cumulus as observed during RICO. J. Adv. Model. Earth Syst., 3, M06001, doi:10.1029/2011MS000056.

Wang, S., B. Albrecht, and P. Minnis, 1993: A regional simulation of marine boundary-layer clouds. J. Atmos. Sci., 50, 4022-4043.

Wyant, M., C. Bretherton, H. Rand, and D. Stevens, 1997: Numerical simulations and a conceptual model of the subtropical marine stratocumulus to trade cumulus. J. Atmos. Sci., 54, 168-192. 